\title{
Interrelation of Precessed Motions of the Gyroscope
}

\author{
Ryspek Usubamatov \\ Kyrgyz State Technical University after I. Razzakov, Bishkek, Kyrgyzstan \\ Email: ryspek0701@yahoo.com
}

How to cite this paper: Usubamatov, $\mathrm{R}$. (2021) Interrelation of Precessed Motions of the Gyroscope. World Journal of Mechanics, 11, 177-185.

https://doi.org/10.4236/wjm.2021.119013

Received: August 24, 2021

Accepted: September 23, 2021

Published: September 26, 202

Copyright (c) 2021 by author(s) and Scientific Research Publishing Inc. This work is licensed under the Creative Commons Attribution International License (CC BY 4.0).

http://creativecommons.org/licenses/by/4.0/

\begin{abstract}
The fundamental principles of the gyroscope theory contain the system of the inertial torques generated by the rotating mass of the spinning disc that interrelated by the ratio of its angular velocities rotation around axes. The action of the centrifugal, common inertial Coriolis forces and the change in the angular momentum generate the system of inertial torques. These four dynamical components make up the system of the eight torques acting simultaneously on the spinning disc. They manifest their action in gyroscopic effects. The ratio of the precessed motions of the gyroscope presents one of the gyroscopic effects around axes of rotation. The known mathematical model for this ratio contains an error that was corrected in this work.
\end{abstract}

\section{Keywords}

Gyroscope Theory, Inertial Torque, Angular Velocity

\section{Introduction}

Gyroscopic effects attract many physicists and mathematicians for over two centuries, as far as researchers cannot find true mathematical models and cannot explain their physics. The term "gyroscopic effects" was derived because of unexplainable motions in space and the action of the inertial forces on the spinning objects [1] [2] [3]. The first scientist that analytically planned only one gyroscopic effect was the famous mathematician L. Euler. He derived the principle of the change in the angular momentum that is the inertial torque acting on the spinning disc. However, this principle could not explain other gyroscopic effects. Beginning with the Industrial Revolution, searchers of all ranks tried to find the analytic approaches and describe the physics of gyroscopic effects related to the many rotating objects in engineering. The formulation of the gyroscope theory 
remained protractedly unsolved until the present time [4] [5] [6].

It is necessary to note that the famous physicists and mathematicians of past times could not develop the gyroscope theory in principle. The chronicle dates of the physical concepts and the properties of the matter are evidence that scientists and researchers of the $17-19$ centuries did not have in full scale the mathematical tools to solve problems of gyroscopic effects [7] [8]. Researchers developed the physical concepts and properties of the matter at different times and could not describe all gyroscopic properties. The fundamental principles of gyroscope theory plan on the action of the system of the inertial torques interrelated by the kinetic ratio of the gyroscope motion around axes of rotation. The scientists of the following centuries could not discover this system of inertial torques acting on the spinning objects in a space [9] [10] [11] [12] [13]. For the practical applications of the gyroscope, they developed complex numerical modeling of gyroscopic effects and simplified mathematical models for the educational processes. The latter ones do not yield correct results [14] [15].

The physics of gyroscopic effects are far more complicated than presented in the simplified theories [1] [2] [3] [4]. The gyroscopic effects express the kinetic energy of the spinning object. The action of the inertial torques and interrelated motions manifest them about the axes of gyroscope rotation. These processes have causal dependencies on the internal torques and their kinetic ratio.

The system of the interrelated inertial torques produced by the external torque $T$ acting on the spinning disc is represented in Figure 1 and Table 1 [16].

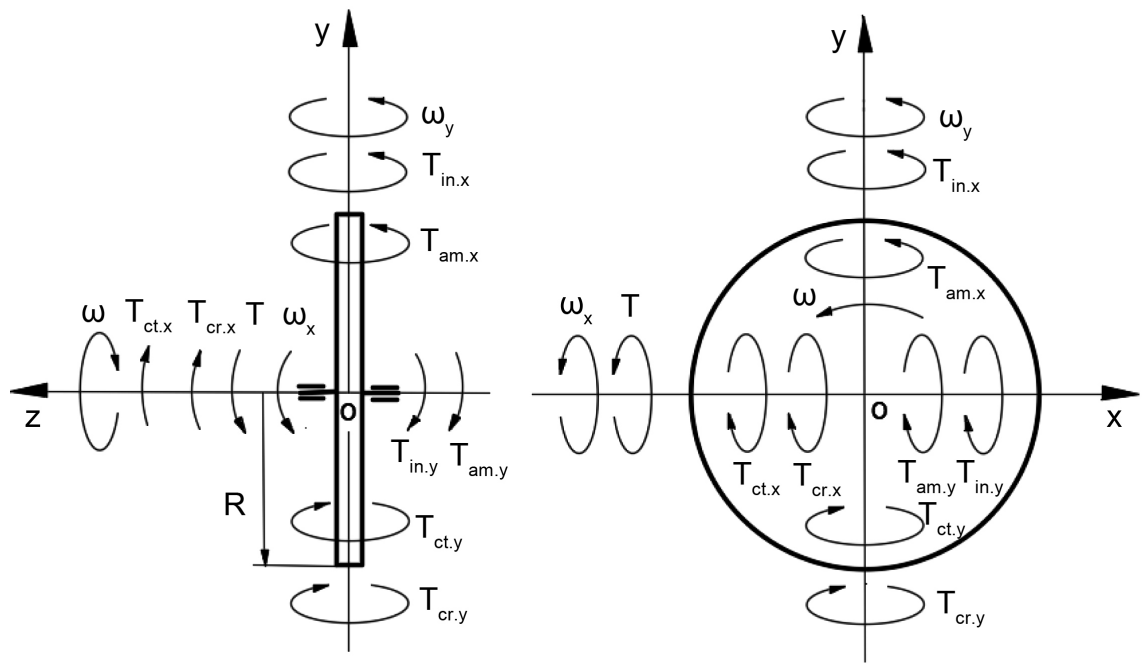

Figure 1. External and inertial torques acting on the spinning disc.

Table 1. Inertial torques acting on the spinning disc of horizontal location.

\begin{tabular}{cc}
\hline Inertial torques acting around axes generated by & Equation \\
\hline Centrifugal forces & $T_{c t . i}=T_{i n . i}=4 \pi^{2} J \omega \omega_{i}$ \\
Inertial forces & $T_{c r . i}=(8 / 9) J \omega \omega_{i}$ \\
Coriolis forces & $T_{a m . i}=J \omega \omega_{i}$ \\
Change in angular momentum
\end{tabular}


Where $\omega_{i}$ is the angular velocity of the spinning disc around axis $i ; \omega$ is the angular velocity of the spinning disc around axis $o Z$, $J$ is the mass moment of inertia of the spinning disc; other components are as specified above.

Practical tests of the action of the external torque on the gyroscope show the different angular velocities of the gimbals around two axes. The minor turn of the external gimbal leads to an intensive turn of the inner gimbal. The gyroscopic effects are a manifestation of the principle of the conservation of mechanical energy for the spinning disc described in publications [17] [18]. The same publications contain proof of the equality of the potential and kinetic energies of the spinning disc around axes of rotation only for its horizontal location. For the angular disposition of the spinning disc, the mathematical model for the kinematic dependency of gyroscope motions around axes is different [16]. The novelty of the presented manuscript is the corrected mathematical model for the ratio of the angular velocities of rotation for the spinning disc around axes. This ratio expresses the kinematic interrelation of the action of the inertial torques generated by the spinning disc.

\section{Methodology}

The kinetic energy of the spinning disc rotation around axes remains constant according to the principle of conservation of mechanical energy. This statement enables the combination of the mechanical energies of the rotating disc around two axes to be described. The inertial torques acting on the spinning disc expresses the kinetic energy of a rotation around axes.

The spinning disc with the inclined axis on the angle $\gamma$ and the action of the external torque (Figure 1) enables expressing the equality of the kinetic energies of the spinning disc that planned by the equality of the inertial torques acting around axes $o x$ and $o y$.

$$
-T_{c t . x}-T_{c r . x}-T_{i n . y}-T_{a m . y}=T_{i n . x} \cos \gamma+T_{a m . x} \cos \gamma-T_{c t . y} \cos \gamma-T_{c r . y} \cos \gamma
$$

where the angle $\gamma$ is the inclination of the disc's axel, other components are as specified in Table 1 and Figure 1.

Substituting expressions of the torques (Table 1) into Equation (1) and transformation yield the following equation:

$$
\begin{aligned}
& -\frac{4 \pi^{2}}{9} J \omega \omega_{x}-\frac{8}{9} J \omega \omega_{x}-\frac{4 \pi^{2}}{9} J \omega \omega_{y}-J \omega \omega \gamma \\
& =\frac{4 \pi^{2}}{9} J \omega \omega_{x} \cos \gamma+J \omega \omega_{x} \cos \gamma-\frac{4 \pi^{2}}{9} J \omega \omega_{y} \cos \gamma-\frac{8}{9} J \omega \omega_{y} \cos \gamma
\end{aligned}
$$

Simplification of Equation (2) yields the following:

$$
\omega_{y}=-\left[\frac{4 \pi^{2}+8+\left(4 \pi^{2}+9\right) \cos \gamma}{4 \pi^{2}+9-\left(4 \pi^{2}+8\right) \cos \gamma}\right] \omega_{x}
$$

where the sign (-) means the direction of the action of the inertial torque that can be omitted from the following analytical considerations. 
Equation (3) presents the variable ratio of the angular velocities for the precessions of the spinning disc around two axes depending on the angle $\gamma$. The ratio $\omega_{y} / \omega_{x}$ of the angular velocities of the gyroscope around axis oy and $o x$ is changed with the change in the angle $\gamma$ (Figure 2).

The diagram of Figure 1 demonstrates the following. The ratio $\omega_{y} / \omega_{x}$ is changed from maximal value for the disc horizontal to zero, with the change in its angular disposition.

For the angle $\gamma=0$ the ratio $\omega_{y} / \omega_{x}$ is maximal as is follows:

$$
\frac{\omega_{y}}{\omega_{x}}=\left[\frac{4 \pi^{2}+8+\left(4 \pi^{2}+9\right) \cos 0}{4 \pi^{2}+9-\left(4 \pi^{2}+8\right) \cos 0}\right]=8 \pi^{2}+17=95.956835
$$

For the turn of the spinning disc axle on the angle, $\varphi_{\max }=168.342302^{\circ}$ the ratio $\omega_{y} / \omega_{x}$ is null, as is follows:

$$
\frac{\omega_{y}}{\omega_{x}}=\left[\frac{4 \pi^{2}+8+\left(4 \pi^{2}+9\right) \cos 168.342302^{\circ}}{4 \pi^{2}+9-\left(4 \pi^{2}+8\right) \cos 168.342302^{\circ}}\right]=0
$$

The turn of the spinning disc around axis $o x$ is active by the ratio $\omega_{y} / \omega_{x}$ until the turn of the spinning disc around axis oy on the angle that is defined by the following expression:

$$
\gamma=\frac{168.342302^{\circ}}{8 \pi^{2}+17}=1.754354^{\circ}
$$

The angles $\gamma=1.754354^{\circ}$ and $\varphi_{\max }=168.342302^{\circ}$ are maximal for the spinning disc turn around two axes simultaneously that maintain their ratio. Practically, the outer gimbal of the gyroscope turns on the angle $\gamma_{\max }=1.754354^{\circ}$ around the vertical axis yields the turn of its internal gimbal with the spinning disc turns on the angle $\varphi_{\max }=168.342302^{\circ}$. The following turn of the outer gimbal does not lead to the turn of the inner gimbal.

Practically, the angular motions of the gyroscopic gimbals are visible on its

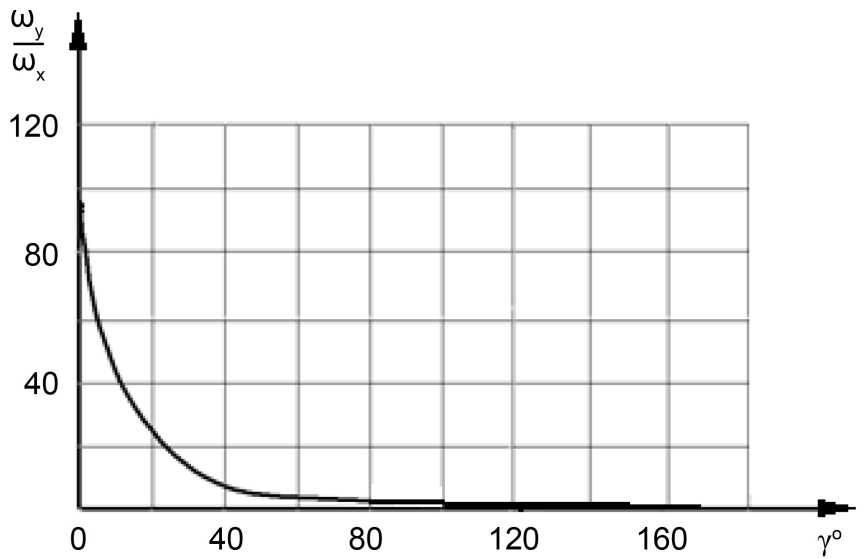

Figure 2. The change of the ratio $\omega_{\mathrm{y}} / \omega_{\mathrm{x}}$ of the angular velocities of the gyroscope around axis $o y$ and $o x$ versus the angle $\gamma$ of the spinning disc turn. 
stand. This gyroscope property is validated by the test of the gyroscope with the horizontal location of the spinning disc axis and the turn of the outer and inner gimbals around axes $o y$ and $o x$. The defined angles of the gyroscope turn around axes are demonstrated on the Super Precision Gyroscope "Brightfusion LTD" (Figure 3).

\section{Case Study and Practical Test}

The interrelated motions of the spinning disc around axes $o x$ and $o y$ result from the action of its inertial torques generated by one rotating mass. The spinning disc with the angular velocity $\omega$, located symmetrically regarding its supports of the gimbal 1, which rotates on supports of the gimbal 2, and later one rotates on pivots of the platform 4 (Figure 3 and Figure 4). The external torque applies to outer gimbal 2 of the gyroscope manifests the motions of the inner gimbal 1 around axis $o x$ of the coordinate $\sum o x y z$. The mathematical model of the ratio for the angular velocities (Equation (3) expresses these motions of the gimbals around axes. The torque $T$ and inertial torques acting on the spinning disc turn the gimbal 2 in the counter-clockwise direction around axis oy (Figure 4(a)). The resulting inertial torques of the spinning disc turns intensively the gimbal 1 in the clockwise direction around axis ox. The turn of the gimbal 2 on the small angle $\gamma=$ around axis $o y$, under the action of the resulting torque, turns the gimbal 1 in the clockwise direction around axis $o x$ on the maximal angle

$\varphi_{\max }=168.342302^{\circ}$ around axis $o x$. The following turn of the gimbal 2 does not turn the gimbal 1 that keeping the vertical location of the spin axis, which coincides with axis oy. Figure 4(b) represents the last location of the gimbals.

The practical test of the gimbal motions was conducted for the horizontal location of the spinning disc and its following turn until vertical. At the starting

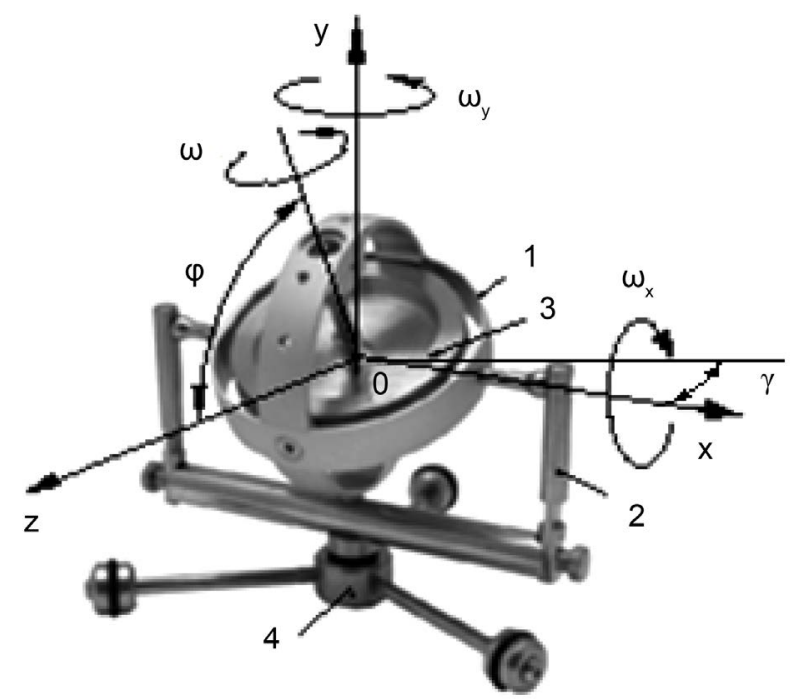

Figure 3. The test stand of super precision gyroscope "Brightfusion LTD". where 1 is the inner gimbal, 2 is the outer gimbal, 3 is the spinning disc, 4 is the platform. 


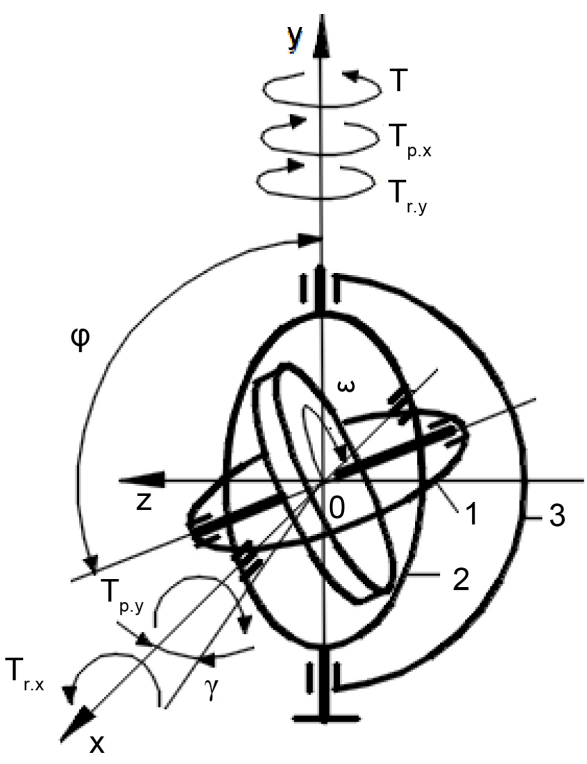

(a)

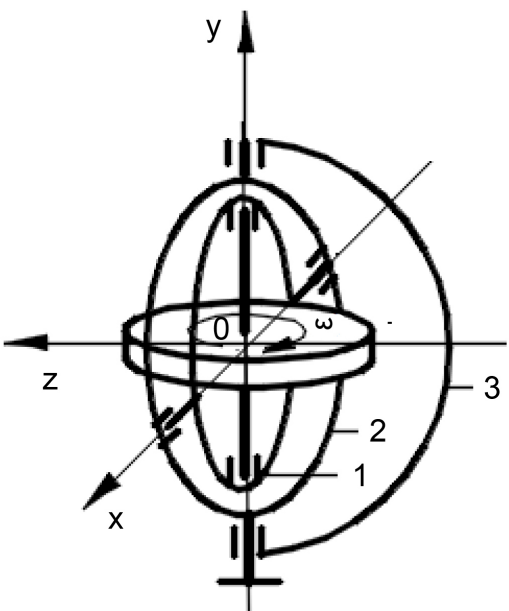

(b)

Figure 4. Motions and locations of the spinning disc under the action of the external torque $T$ applied to the gimbal.

condition, the disposition of the spinning disc and the outer gimbal is showed in Figure 5(a). The minor turn of the outer gimbal in the counter-clockwise direction around the vertical axis of the platform leads to the intensive turn of the inner gimbal in the clockwise direction around the horizontal axis (Figure 5(b)). The inner gimbal turns until the vertical location of the spinning disc axis with the minor turn of the outer gimbal on the angle $\gamma$ (Figure $5(\mathrm{c})$ ). The following turn of the outer gimbal does not turn the inner gimbal (Figure 5(d)).

The Mitutoyo Universal Bevel Protractor was used to conduct the measurement of the angles of the turn for the gimbals. The angle of the turn of the inner gimbal on $\omega_{y} t=90^{\circ}$ from horizontal to vertical location implements by the minor turn of the outer gimbal on the angle $\omega_{x} t=\gamma$ that defined by Equation (3).

$$
90^{\circ}=\left[\frac{4 \pi^{2}+8+\left(4 \pi^{2}+9\right) \cos 0^{\circ}}{4 \pi^{2}+9-\left(4 \pi^{2}+8\right) \cos 0^{\circ}}\right] \gamma \text { and } \gamma=0.937921^{\circ}=0^{\circ} 56^{\prime} 16^{\prime \prime}
$$

The measurements of the angle $\gamma=0.937921^{\circ}$ were problematic due to the high sensitivity on the manual turn of the outer gimbal that yields the turn of the inner gimbal on $90^{\circ}$. The obtained result of practical motions of the gimbals is the validation of the correctness of Equations (4)-(6) that matched theoretical results. The turn of the inner gimbal around axis $o x$ on $\varphi_{\max }=168.342302^{\circ}$ was confirmed approximately because the measurement by the tool was problematic due to the constructional properties of the gyroscope cage. Figure 6 shows the change in the angular disposition of the inner gimbal versus the change in the angular location of the outer gimbal. The results have explained the physics of gyroscopic gimbal motions that were one in the series of unsolved gyroscopic effects. 


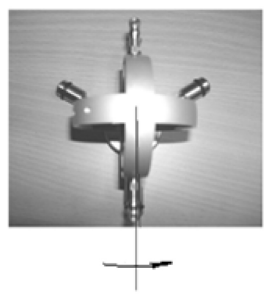

(a)

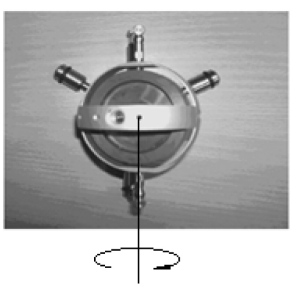

(b)

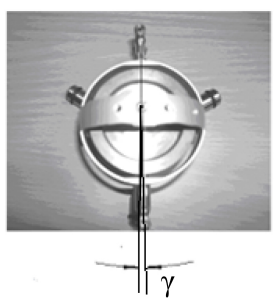

(c)

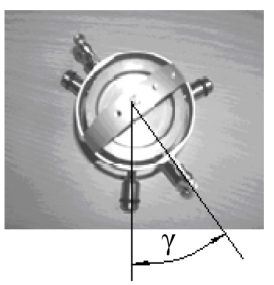

(d)

Figure 5. Disposition of the spinning disc with the inner gimbal at the process of the turn of the outer gimbal.

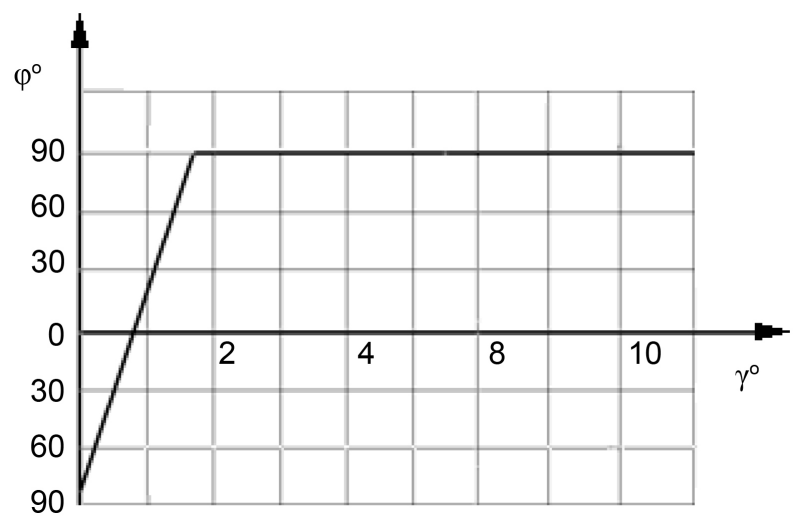

Figure 6. The angular location of the outer gimbal versus the angular location of the spinning disc and the inner gimbal.

The mathematical model for the ratio of the angular velocities of gyroscope motions describes their physics. These gyroscope properties can be useful for modeling the behavior for the gyroscopic devices that is routing work for practitioners.

\section{Results and Discussion}

The planned fundamental principles of the theory of gyroscopic effects contain minor inevitable errors of the first edition. The author should correct the gyroscope theory to avoid criticism of users. One of the mathematical errors of the theory of gyroscopic effects is the ratio of the precessed motions of the gyroscope around axes of rotation. This error results from the incomplete presentation of the angular motions of the spinning disc around axes of rotation. The corrected ratio of the angular velocities of the gyroscope around axes of rotation is observed and confirmed practically.

\section{Conclusion}

The theory of gyroscopic effects for spinning objects opens a new direction in classical mechanics for computing inertial forces and motions of the objects in space. Science and engineering receive a new powerful analytical tool and methods that enable solving gyroscopic effects in engineering. The first publication of the theory of gyroscopic effects contains some errors in the analytical formulation of 
the acting inertias tortures that must be corrected for sake of wide use in practice. One error in the mathematical model for the ratio of the precessed motions of the gyroscope around axes of rotation is corrected and can be used for many mechanical gyroscopic devices in engineering.

\section{Conflicts of Interest}

The author declares no conflicts of interest regarding the publication of this paper.

\section{References}

[1] Cordeiro, F.J.B. (2015) The Gyroscope. CreateSpace, Nevada.

[2] Greenhill, G. (2015) Report on Gyroscopic Theory. Relink Books, Fallbrook.

[3] Scarborough, J.B. (2014) The Gyroscope Theory and Applications. Nabu Press, London.

[4] Weinberg, H. (2011) Gyro Mechanical Performance: The Most Important Parameter. Technical Article MS-2158, Analog Devices, Norwood, 1-5.

[5] Hibbeler, R.C. and Yap, K.B. (2013) Mechanics for Engineers-Statics and Dynamics. 13th Edition, Prentice Hall, Pearson, Singapore.

[6] Gregory, D.R. (2006) Classical Mechanics. Cambridge University Press, New York.

[7] Taylor, J.R. (2005) Classical Mechanics. University Science Books, California.

[8] Aardema, M.D. (2005) Analytical Dynamics. Theory and Application. Academic/ Plenum Publishers, New York.

[9] Liang, W.C. and Lee, S.C. (2013) Vorticity, Gyroscopic Precession, and Spin-Curvature Force. Physical Review D, 87, Article ID: 044024.

https://doi.org/10.1103/PhysRevD.87.044024

[10] LeMoyne, R., Mastroianni, T., McCandless, C., Currivan, C., Whiting, D. and Tomycz, N. (2018) Implementation of a Smartphone as a Wearable and Wireless Accelerometer and Gyroscope Platform for Ascertaining Deep Brain Stimulation Treatment Efficacy of Parkinson's Disease through Machine Learning Classification. Advances in Parkinson's Disease, 7, 19-30.

[11] Yong, C.Y., Sudirman, R., Mahmood, N.H. and Chew, K.M. (2013) Motion Classification Using Proposed Principle Component Analysis Hybrid K-Means Clustering. Engineering, 5, 25-30. https://doi.org/10.4236/eng.2013.55B006

[12] Crassidis, J.L. and Markley, F.L. (2016) Three-Axis Attitude Estimation Using RateIntegrating Gyroscopes. Journal of Guidance, Control, and Dynamics, 39, 1513-1526. https://doi.org/10.2514/1.G000336

[13] Nanamori, Y. and Takahashi, M. (2015) An Integrated Steering Law Considering Biased Loads and Singularity for Control Moment Gyroscopes. AIAA Guidance, Navigation, and Control Conference, Kissimmee, 5-9 January 2015. https://doi.org/10.2514/6.2015-1091

[14] Jewett, J. and Serway, R.A. (2018) Physics for Scientists and Engineers. 10th Edition, Cengage Learning, Boston.

[15] Knight, R.D. (2016) Physics for Scientists and Engineers: A Strategic Approach with Modern Physics. 4th Edition, Pearson, London.

[16] Usubamatov, R. (2018) Inertial Forces Acting on Gyroscope. Journal of Mechanical Science and Technology, 32, 101-108. https://doi.org/10.1007/s12206-017-1211-0 
[17] Usubamatov, R. (2015) Mathematical Model for Gyroscope's Gimbal Motions. 4th International Conference on Advances in Engineering Sciences \& Applied Mathematics (ICAESAM2015), Kuala Lumpur, 8-9 December 2015, 41-44.

[18] Usubamatov, R. (2020) Theory of Gyroscope Effects for Rotating Objects. Springer, Singapore. https://doi.org/10.1007/978-981-15-6475-8 